\title{
Network tackles overprescribing
}

$\mathrm{D}$ octors need better information and support from other health professionals to wean patients from unnecessary medications, say experts from the Canadian Deprescribing Network (CaDeN).

"The pendulum has swung too far and there is too much chemical coping," says geriatrician Cara Tannenbaum, the codirector of $\mathrm{CaDeN}$ - the network of physicians, pharmacists and others formed last year to coordinate research on curbing prescription drug use among seniors.

Nearly $70 \%$ of Canadians over 65 take five or more medications and almost $10 \%$ take 15 or more, putting them at higher risk of harm from side effects. Seniors often end up in hospital as a result of bad reactions to medications, and it's estimated that most of these cases are predictable and potentially avoidable.

Recent studies have shown that deprescribing is safe, but Tannenbaum says many doctors lack the training or confidence to identify and stop potentially inappropriate prescriptions. "Pharmacists take four years of school to figure this out, and physicians get 10 minutes at the end of the hour on arthritis, for example, about what pills to use."

Prescribing decisions are more complicated when it comes to seniors because drug information usually comes from studies of younger, healthier patients who are taking no other medications, she explains.

According to Barbara Farrell, a pharmacist and CaDeN's other codirector, clinical guidelines aren't much help either: "They only recommend starting drugs, never stopping them."

"If you have a patient on 20 medications, you might not want to stop one without looking at the rest, and the expertise to do that is just overwhelming."

Farrell led the recent development of deprescribing guidelines for three drugs that are often overprescribed to seniors: proton pump inhibitors, benzodiazepines and antipsychotics.

A pilot of the guidelines at six practice sites in Ottawa showed that doctors were more likely to use the advice if they worked in teams with pharmacists and nurse practitioners.
Another ongoing study presented at the national Choosing Wisely Canada Conference in March showed that pharmacists can also support deprescribing by sending letters to doctors with recommendations for stopping or reducing potentially inappropriate prescriptions.

"We offered all kinds of alternatives, as well as a checkbox for making the prescription official," explained lead investigator Philippe Martin. Half the doctors responded to the letters and, in more than half the cases, stopped or significantly lowered the targeted prescriptions, he said. The full results of the trial will be available in 2017.

Farrell says recent provincial projects to embed pharmacists in family practice clinics are a step in the right direction to share the burden of deprescribing. "We also need to change the way the system works, for example, to compensate those two to talk to each other."

Other health professionals also have important roles to play in deprescribing, she adds. For example, an occupational therapist could give patients advice about walking aids to reduce reliance on pain medication, or a dietitian could help patients change what they eat to reduce their need for stomach medications.

Limited funding for these alternative treatments is a factor in the difficulty of deprescribing, Farrell says. "Cognitive behavioural therapy has proven as effective as sleeping pills in the treatment of insomnia, but we don't cover psychologist or social worker care for that, so it can be very expensive."

Doctors also avoid raising the issue because of concerns about how their patients will react, says family physician and primary care researcher Dr. MarieThérèse Lussier. "I think it's probably something that we as doctors might exaggerate."

In her ongoing qualitative study of deprescribing conversations, Lussier has found that patients are actually quite open to stopping medications if their doctors suggest it. "They feel confidence that their doctor is doing this for their own good, and we haven't heard any patients questioning why the drug was prescribed in the first place." - Lauren Vogel, CMAJ

CMAJ 2016. DOI:10.1503/cmaj.109-5315 